\title{
Review \\ The Role of Bacteria in KSHV Infection and KSHV-Induced Cancers
}

\author{
Ashley Markazi ${ }^{1,2}$, Wen Meng ${ }^{1,2}$, Paige M. Bracci ${ }^{3}$, Michael S. McGrath ${ }^{4}$ and Shou-Jiang Gao 1,2,* \\ 1 Cancer Virology Program, UPMC Hillman Cancer Center, Pittsburgh, PA 15213, USA; \\ admarkazi@gmail.com (A.M.); mengw@upmc.edu (W.M.) \\ 2 Department of Microbiology and Molecular Genetics, University of Pittsburgh School of Medicine, \\ Pittsburgh, PA 15219, USA \\ 3 Department of Epidemiology and Biostatistics, University of California at San Francisco, \\ San Francisco, CA 94158, USA; paige.bracci@ucsf.edu \\ 4 Department of Laboratory Medicine, Pathology and Medicine, University of California at San Francisco, \\ San Francisco, CA 94143, USA; Mike.McGrath@ucsf.edu \\ * Correspondence: gaos8@upmc.edu
}

Citation: Markazi, A.; Meng, W.; Bracci, P.M.; McGrath, M.S.; Gao, S.-J. The Role of Bacteria in KSHV Infection and KSHV-Induced Cancers. Cancers 2021, 13, 4269. https:/ / doi.org/10.3390/cancers13174269

Academic Editor: David Wong

Received: 17 July 2021

Accepted: 19 August 2021

Published: 25 August 2021

Publisher's Note: MDPI stays neutral with regard to jurisdictional claims in published maps and institutional affiliations.

Copyright: (c) 2021 by the authors. Licensee MDPI, Basel, Switzerland. This article is an open access article distributed under the terms and conditions of the Creative Commons Attribution (CC BY) license (https:// creativecommons.org/licenses/by/ $4.0 /)$.
Simple Summary: The aim of this article is to review the complex interactions of bacteria with Kaposi's sarcoma-associated herpesvirus (KSHV) infection and KSHV-induced cancers. KSHV is causally associated with multiple cancers including Kaposi's sarcoma (KS) and primary effusion lymphoma. Among patients coinfected by HIV and KSHV, patients with KS have a distinct oral microbiome compared to patients without KS. Moreover, KSHV patients have increased levels of salivary bacterial pathogen-associated molecular patterns compared to KSHV-negative patients. KSHV-associated bacterial species can increase KSHV replication and dissemination, and enhance cell proliferation of KSHV-transformed cells. The analysis of bacterial biomarkers associated with KSHV may help improve our understanding of the mechanisms driving KSHV-induced oncogenesis and identify novel targets for improving therapies of KSHV-related cancers.

Abstract: The objective of this article is to review the current status of the bacteria-virus interplay in Kaposi's sarcoma-associated herpesvirus (KSHV) infection and KSHV-driven cancers. KSHV is the etiological agent of several cancers, including Kaposi's sarcoma (KS) and primary effusion lymphoma. Due to immunosuppression, patients with KSHV are at an increased risk for bacterial infections. Moreover, among patients coinfected by HIV and KSHV, patients with KS have distinct oral microbiota compared to non-KS patients. Bacterial biomarkers associated with KSHV-driven cancers can provide insights in discerning the mechanisms of KSHV-induced oncogenesis. For example, pathogen-associated molecular patterns and bacterial products of certain bacterial species can regulate the expression of KSHV lytic and latent genes, thereby affecting viral replication and dissemination. In addition, infection with distinct opportunistic bacterial species have been associated with increased cell proliferation and tumorigenesis in KSHV-induced cancers through activation of pro-survival and -mitogenic cell signaling pathways. By elucidating the various mechanisms in which bacteria affect KSHV-associated pathogenesis, we will be able to pinpoint therapeutic targets for KSHV infection and KSHV-related cancers.

Keywords: Kaposi's sarcoma; KSHV; microbiome; HIV; opportunistic infection

\section{Introduction}

The utilization of bacterial markers to determine cancer pathology has progressed tremendously in recent years. Advances in technology have empowered researchers to more thoroughly elucidate complex interactions between the microbiome and cancer. Microbiome analyses using next generation sequencing report that the colonization of pathogenic bacterial species is increased in cancer patients and exacerbates cancer pathogenesis [1]. Pathogenic bacteria such as Fusobacterium nucleatum and Helicobacter pylori 
have been extensively studied for their carcinogenic effects, as they can produce toxins and effector proteins that induce host cell damage and alter cell signaling pathways involved in cell survival and proliferation [2]. Contrarily, many studies have shown that certain bacteria such as probiotics have anti-inflammatory effects that may aid in cancer prevention [3]. Moreover, recent studies have shown that bacteria can interact with cancer-causing viruses to promote viral replication and the progression of viral cancers [4]. The analysis of bacteria-virus interactions is paramount to microbiome-cancer studies, as up to $15 \%$ of cancers have a viral etiology [5]. This review will specifically focus on the effects of bacteria on infection of Kaposi's sarcoma-associated herpesvirus (KSHV) and its associated cancers, which develop most frequently in immunosuppressed individuals. To thoroughly comprehend the interactions between bacteria and KSHV-induced cancers, it is necessary to begin with a brief overview of the general interactions between the microbiome, immune response, and cancer.

\section{Bacterial Infection and the Innate Immune Response: Effects on Cancer Pathology}

The human immune system responds to bacterial infection by producing inflammation. Bacteria possess pathogen-associated molecular patterns (PAMPs) that are recognized by pattern recognition receptors (PRRs) expressed by both non-immune and immune cells [6,7]. Activation of PRRs causes a downstream cascade of signal transduction pathways, resulting in enhanced expression of inflammatory cytokines [6]. Inflammatory cytokines amplify inflammation by signaling additional immune cells to migrate towards the infection [6]. Immune cells, in addition to producing more cytokines, respond to infection by generating antibacterial substances including reactive oxygen species (ROS) or reactive nitrogen species (RNS), which can cause apoptosis in infected cells [8]. Bacterial infection can also activate the complement system, resulting in induction of inflammatory mediators, increased angiogenesis, and migration of immune cells to the infection [9]. Although inflammation is necessary for controlling infection, unregulated or continuous inflammation can initiate and/or increase carcinogenesis [10].

A number of studies have annotated the pathways linking PRR activation and cancer cell proliferation [11]. Bacterial PAMPs can activate transmembrane and cytosolic PRRs [6]. Transmembrane receptors include C-type lectins receptors and toll-like receptors (TLRs) that recognize extracellular and endosomal-derived PAMPs [12,13]. Cytosolic receptors include nucleotide-binding domain leucine-rich repeat containing receptors (NLRs) and RIG-I like receptors (RLRs) that recognize intracellular infections and damage-associated molecular patterns (DAMPs) [6]. Activation of these PRRs causes induction of inflammatory pathways including the NF-kB pathway and mitogen activated protein kinase (MAPK) pathways which increase expression of cytokines such as IL-10, TNF- $\alpha$, IL-1 $\beta$, and IL-6 [6]. The oldest of PRRs are the TLRs, which recognize bacterial structure components. For examples, lipopolysaccharides (LPS) are detected by TLR4, lipoproteins are detected by TLR1/2 or TLR2/ 6 heterodimers, and flagellin is detected by TLR5 [6]. Studies have demonstrated that bacterial ligands including LPS, peptidoglycans, and flagellin promote cell proliferation and/or invasiveness in several cancer cell lines [14-18]. Moreover, some studies suggest that increased levels of circulating bacterial PAMPs are correlated with a higher cancer risk [19-21].

However, contradictory studies show decreased cell proliferation in cells stimulated with bacterial PAMPs [22-24]. Moreover, bacteria and their PAMPS have been studied as potential therapies for decreasing tumors $[25,26]$. These contradictory effects are likely due to the multiple responses of cells to TLR activation or differences within the tumor environment (such as compositions of immune cells) [22,27-29]. Elucidating the factors that direct the downstream signaling of TLRs toward pro-survival versus apoptotic pathways will be especially important for determining how to best utilize bacteria for cancer therapy.

Due to the substantial evidence of bacterial impact on cancer pathology, it is essential to delineate the intricate interactions of the bacterial microbiome with cancer. The microbiome consists of the microbial community, which maintains intricate interactions. Their 
effects on the habitat are complex [30]. The human body is home to a multitude of different types of microbiomes, each with a distinct composition of microbial species [31]. The intestines and oral cavities harbor the greatest number and diversity of species [31]. Cancerous tumors and lesions also have distinct microbiomes, consisting of intracellular bacterial species highly specific to the particular tumor type [32]. The species' composition and bacterial interactions dictate the types of bacterial metabolites, biofilm, and quorum signaling molecules produced within the microbiome [30]. In addition to being parts of the bacterial structures, bacterial products have a significant effect on immune response [33]. The bacterial microbiome can vary greatly based on many genetic and environmental factors, including the host's diet, location, weight, and ethnicity [34]. Based on the current literature, microbial effects on cancer can be broadly classified into three major categories: (1) dysregulation of host immunity induced by bacterial PAMPs, (2) direct interactions between bacterial products and cancer cells, (3) indirect mechanisms involving bacterial products, and (4) for viral cancers, direct interactions between bacteria and bacterial products, and viruses.

\section{KSHV and KSHV-Associated Cancers}

This review focuses on the effects of bacteria on KSHV infection and KSHV-associated cancers. KSHV is the etiological agent of Kaposi's sarcoma (KS) and primary effusion lymphoma (PEL) [35]. Although KSHV infection has higher prevalence in the homosexual population, infection rates vary in the general population according to geographic regions [36]. Its infection rate is the highest in some African regions (reaching 70\%), median in Eastern European and Mediterranean regions (in the range of 20-30\%), and lowest in North America and most other European and Asian countries (in the range of 4-12\%) [37]. Importantly, most individuals with KSHV infection do not develop any KSHV-associated cancers, implying that additional co-factors are necessary for KSHV-infected subjects to develop cancer [38].

As a gammaherpesvirus, KSHV causes life-long persistent infection [39]. The KSHV latency phase is characterized by the expression of limited viral latent genes that helps the virus escape from the host's immune detection [40]. The lytic phase consists of highly orchestrated expression of viral lytic genes that lead to viral DNA replication and virion release [40]. Lytic replication is essential for both transmission and dissemination of the virus [40]. The majority of viral genes are lytic and are silenced during latency through multiple epigenetic modifications of the viral genome including DNA methylation, and histone deacetylation and repressive histone methylation [40]. Physiological factors that activate KSHV lytic replication include immunosuppression, hypoxia, inflammation, and copathogenic infections [4].

Since not all individuals infected with KSHV develop malignancies, it is especially important to understand the roles co-factors play in the development and severity of KSHV-induced cancers. KSHV most commonly causes cancers in immunocompromised individuals, especially those with HIV infection [35]. Patients with HIV have decreased CD4+ T cell counts, which plays a crucial role in controlling KSHV lytic replication and KS tumors [41,42]. HIV proteins TAT (trans-activator of transcription), Nef, and Vpr can directly interact with and regulate the functions of KSHV proteins in addition to altering host immune regulation, leading to promotion of KSHV infection [43-48]. Studies have shown that low CD4+ T cell count may increase the risk of developing KS and PEL, further indicating the significance of immunosuppression on KSHV-induced pathologies [4].

Suppressed immunity associated with HIV increases the risk of opportunistic infections [4]. Several studies show that microbiomes of immunosuppressed patients have a lower overall diversity of bacterial species and increased colonization of pathogenic species compared to healthy individuals $[49,50]$. Oral bacterial infection, in particular, is highly relevant in KS patients, as periodontal disease is present in higher frequency in KS patients than healthy individuals [4]. Gruffaz et al. used next generation sequencing to analyze the microbiota in saliva samples of KS patients with either oral KS, no oral KS 
but oral cell-associated KSHV, or neither oral KS nor oral cell-associated KSHV DNA [51]. Oral KS patients had the most phylogenetically distant composition of bacteria compared with the two other groups [51]. Figure 1 depicts the observed increases and decreases of oral microbiota in the oral KS group compared with the HIV/KSHV-coinfected patients without oral KS or oral KSHV [51]. Oral KS patients had higher levels of the phyla Firmicutes and Actinobacteria and decreased levels of the phyla Bacteroidetes and Proteobacterium. Moreover, oral KS patient samples had higher levels of common oral pathogens including bacteria in the genera Corynebacterium and Shuttleworthia [51]. These results strongly imply that oral microbiota interaction with HIV/KSHV coinfection may play an important role in influencing the development of oral KS. Other studies have also detected a positive correlation between herpesvirus and periodontal pathogens, including Porphyromonas. gingivalis and Fusobacterium nucleatum [52]. Moreover, many studies have reported that common oral pathogens produce metabolites that reactivate KSHV and Epstein-Barr virus (EBV), another oncogenic gammaherpesvirus, resulting in enhanced viral infectivity and promotion of lytic replication of the virus [53-55].

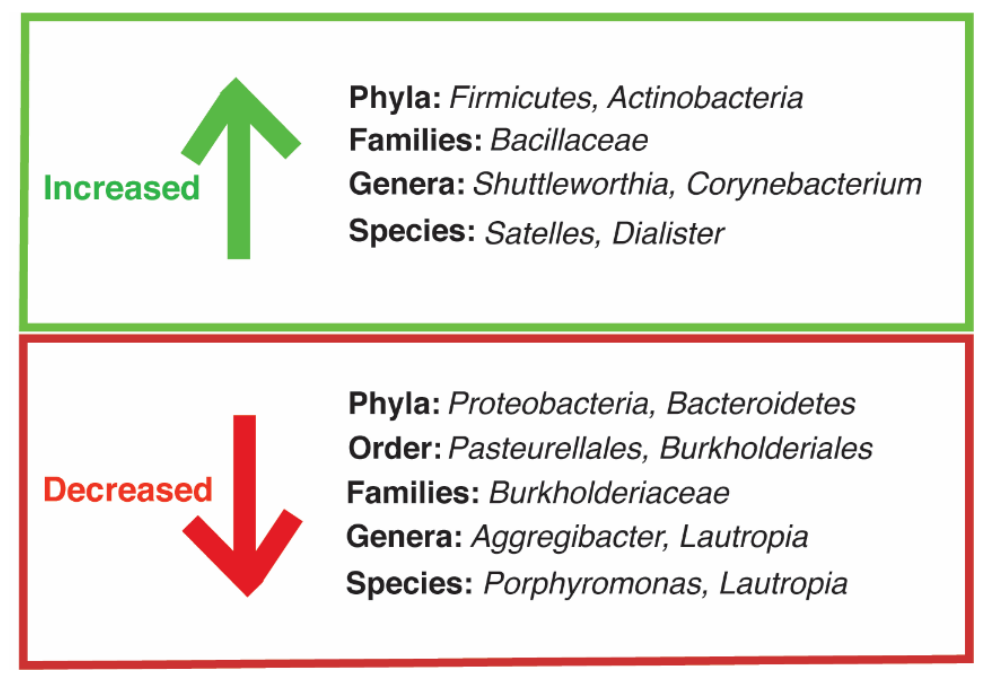

Figure 1. Observed increases and decreases in phyla, orders, families, genera, and species of oral microbiota in HIV/KSHV-coinfected oral KS patients compared with HIV/KSHV-coinfected patients without oral KS or oral KSHV. Figure was modified from Figure 5 in the open access article by Gruffaz et al. [51].

\section{Impact of Bacteria on KSHV Lytic Replication}

Butyrate, a common metabolic end product of certain bacterial species, has been shown to reactivate herpesviruses including KSHV [55]. Butyrate inhibits the activities of histone deacetylases (HDACs) and promotes hyperacetylation, leading to increased viral gene expression [56]. Multiple studies have shown that the medium of butyrate-producing oral pathogens can result in KSHV reactivation [53,55]. A study by Morris et al. explored the role of microbial infection in KSHV reactivation by treating PEL cell line BCBL1 cells with spent medium from oral disease pathogens including $P$. gingivalis, F. nucleatum, Prevotella intermedia, and Streptococcus mutans [55]. Authors detected increased viral lytic gene expression in cells treated with mediums from the potent butyrate-producing bacteria, namely the $P$. gingivalis and $F$. nucleatum medium. The study also examined the mechanism of bacterial-induced reactivation and reported that activation of viral lytic genes by the bacterial mediums occurred through the p38 MAPK pathway as this pathway has been shown to be both essential and sufficient for KSHV lytic replication during primary infection and reactivation [55,57-60].

Yu et al. further explored the effects of medium from F. nucleatum and P. gingivalis on KSHV reactivation [53]. Their study reported increased expression of viral lytic gene expression in BCBL1 cells treated with medium from P. gingivalis and F. nucleatum. More- 
over, treatment of BCBL1 cells with different doses of pure individual short chain fatty acids (SCFAs) detected in the mediums of $P$. gingivalis and F. nucleatum except acetic acid resulted in increased expression of lytic gene RTA (ORF50) [53]. Treatment with butyric acid resulted in the greatest effect, whereas an additive effect was observed when SCFAs were combined [53]. Interestingly, by analyzing levels of SCFAs in saliva, Yu and colleagues found higher concentrations of salivary SCFAs from $P$. gingivalis and $F$. nucleatum in patients with periodontal diseases compared to healthy controls [53]. Yu et al. explored the mechanism of KSHV reactivation by bacteria, reporting that BCBL1 cells, KSHV-infected human normal epithelial cells (HOECs), and KSHV-infected human telomerase-immortalized human umbilical vein endothelial cells (TIVE-KSHV) treated with P. gingivalis and F. nucleatum had significantly reduced expression of class $1 / 2$ HDACs. In contrast, no effect was seen with Escherichia coli medium [53]. These findings provide evidence that SCFAs from periodontal pathogens might reactivate KSHV in the oral cavity and therefore influence the development and progression of KSHV-induced cancers.

Besides SCFA production, additional bacterial effects have been linked to KSHV reactivation, including inflammation related to cytokines and ROS [42]. Figure 2 depicts bacteria-related factors that have been shown to reactivate KSHV. Ye et al. observed that both exogeneous and endogenous hydrogen peroxide $\left(\mathrm{H}_{2} \mathrm{O}_{2}\right)$ increased KSHV lytic gene expression in HUVEC and PEL cell lines [61]. Although, at higher concentrations, $\mathrm{H}_{2} \mathrm{O}_{2}$ causes apoptosis and senescence in primary cells, at lower concentrations, $\mathrm{H}_{2} \mathrm{O}_{2}$ can activate multiple redox signaling pathways such as the MAPK pathways [61]. Ye et al. reported that $\mathrm{H}_{2} \mathrm{O}_{2}$ induction of KSHV reactivation depended on ERK1/2, JNK, and p38 pathways [61]. As $\mathrm{H}_{2} \mathrm{O}_{2}$ can be produced directly by bacteria and indirectly by bacterial stimulation of immune cells, it may be of interest to more thoroughly assess the impact of bacterial infection in causing KSHV reactivation through this mechanism [62].

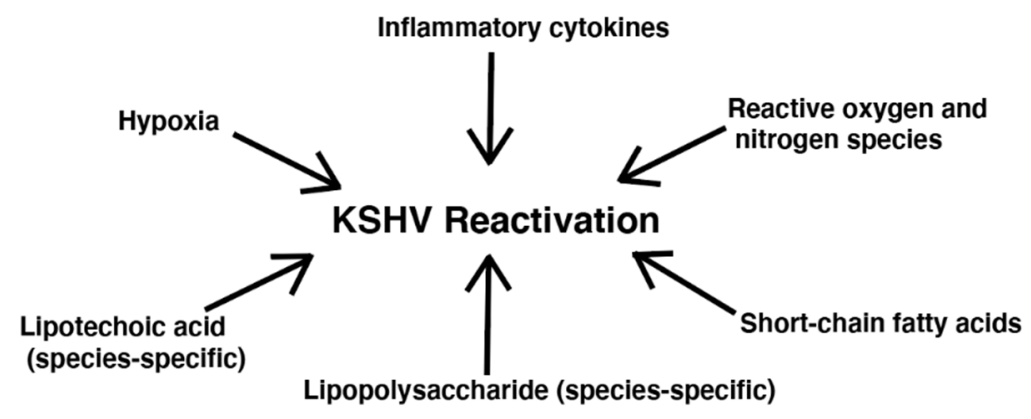

Figure 2. Bacteria-related factors that reactivate KSHV.

Dai et al. reported that both bacterial PAMPs from S. aureus and P. gingivalis influenced KSHV entry into cells and subsequent expression of viral genes in primary human gingival fibroblasts (HDF) and periodontal ligament fibroblasts (PDLF) [63]. Dai et al. further explored if ROS was related to LTA/LPS facilitation of KSHV replication in oral cells [63,64]. They indeed found that LTA/LPS treatment significantly increased intracellular ROS production and NADPH oxidase activity in HGF and PDLF [63]. These results imply that bacteria might be the inducer of ROS production, and regulate KSHV lytic replication. Furthermore, LTA from $S$. aureus induced MAPK-ERK phosphorylation and LPS from P. gingivalis increased NF- $\mathrm{kB}$ phosphorylation in HGF [63]. It has previously been reported that once KSHV enters the cell, both the NF- $\mathrm{KB}$ and MAPK signaling pathways are required for successful establishment of latent infection $[65,66]$. However, in PDLF cells, both LTAand LPS-induced NF- $\mathrm{KB}$ P65 had little effect on the MAPK pathways [63]. It is therefore apparent that the mechanism of PAMP-induced viral entry requires further investigation. The study did not look at the effects of TLR activation, which may be relevant as LTA and LPS are prominent ligands for TLR2 and TLR4, respectively. However, these results show that the microbiome in the oral cavity provide an amenable setting for KSHV infection and promote virus dissemination. 
Dai et al. also examined the effects of $S$. aureus on KSHV reactivation [67]. S. aureus is a common, gram-negative intracellular pathogen that can colonize in the oral cavity of patients with periodontitis [68]. In both human gingival and periodontal ligament fibroblasts treated with the $S$. aureus medium, there were increased expression of KSHV lytic genes including RTA, vGPCR (ORF74), ORF-K8.1, and ORF57 [67]. Treatment with the $S$. aureus medium also induced release of infectious virion particles from both the gingival fibroblasts and PDLF. However, no significant effects from the medium of other gram-positive bacteria tested, such as Bacillus subtilis were observed, suggesting specific effect of the bacterial species. HIV $+/ \mathrm{KSHV}+$ patients also had higher levels of salivary LTA, a bacterial ligand for TLR2. However, the LTA ELISA kit used to analyze LTA levels did not distinguish LTA levels among different bacterial species. It would therefore be of interest to examine the effects of LTA from different gram-positive species on KSHV reactivation [67].

Mechanistically, Dai et al. found that $S$. aureus medium reduced the expression of KSHV microRNAs (miRNAs) compared with cells treated with control and fresh medium [67]. KSHV miRNAs play an important role in maintaining viral latency [67]. Furthermore, S. aureus medium downregulated expression of cyclin D1 and other host proteins responsible for processing cellular miRNAs, including Dicer, and Argonaut 1 and 2. These results suggested that the $S$. aureus medium might induce KSHV reactivation through the cyclin D1-Dicer-viral miRNAs axis in KSHV-infected oral cell lines [67]. The study also analyzed the effect of $S$. aureus and KSHV coinfection since $S$. aureus is an intracellular pathogen that can invade and survive in many cells including fibroblasts and endothelial cells. The authors concluded that coinfection in the same single cells induced KSHV reactivation. Moreover, a bacterial analysis of saliva samples in HIV+ patients showed $72 \%$ S. aureus compared to $37 \%$ in HIV-subjects [67]. This study is clinically relevant since methicillin-resistant S. aureus (MRSA) infection is a common complication in HIV patients [69]. Due to the ubiquitous nature of S. aureus, it may be worthwhile to investigate the effects of $S$. aureus in additional sites other than the saliva in HIV/KSHV-coinfected patients. Moreover, this study examined the conditioned medium of $S$. aureus which might contain many bacterial products besides LTA. It may be important to study the effects of purified LTA on KSHV-infected cells.

In a separate study, Dai et al. analyzed saliva samples for the presence of $P$. gingivalis and KSHV infection in HIV+ patients, and found that $11.3 \%$ were positive for both P. gingivalis (a periodontitis-associated bacterium) and KSHV [54,70]. There were also increased salivary LPS levels in KSHV-coinfected patients compared to patients without KSHV infection [54]. Mechanistically, Dai et al. found the P. gingivalis medium and LPS increased the expression of KSHV lytic genes in latently infected primary human oral fibroblasts through TLR4 [54]. However, authors suggested that additional bacterial products in the medium besides LPS might induce viral lytic gene expression, as blocking TLR4 expression did not completely abrogate the effect of $P$. gingivalis [54]. Likewise, $P$. gingivalis medium and LPS activated the MAPK pathways in KSHV-infected oral cells by inducing phosphorylation of p38 and JNK. However, treatment with p38 or JNK inhibitors did not completely hinder viral lytic reactivation, implying other pathways may be involved [54]. Interestingly, no significant effects were observed using medium or LPS from E. coli [54]. The differences in species-specific effects of LPS on KSHV reactivation is worth looking into. It is possible that the differences may be due to different LPS structures between the bacteria. The structure of the O-antigen as well as acylation patterns of the LPS structure may cause differences in receptor activation [71].

\section{Impact of Bacteria on KSHV-Induced Cell Proliferation and Cellular Transformation}

While lytic activation of KSHV is necessary for disseminating the infection, latency is essential for establishing persistent KSHV infection [40]. Upon KSHV infection, most cells remain in the latent state [72]. During latency, the virus expresses minimal proteins to evade the host immune response [40]. Epigenetic modifications of the viral genome and 
its associated histones play a crucial role in silencing viral lytic genes during latency [73]. Despite silenced expression of viral lytic proteins during latency, latent proteins are expressed resulting in cell proliferation by activating cell signaling pathways such as the STAT3 pathway, which is constitutively active in KSHV-infected cells [74,75]. Furthermore, during latency, KSHV utilizes the complement pathway to increase cell survival [76]. As the complement system has a central role in the defense against pathogens, it is particularly relevant to assess the bacterial effects on KSHV latently-infected cells in addition to lytic reactivation [9].

Gruffaz et al. analyzed the expression of TLRs in rat primary embryonic metanephric mesenchymal precursor (MM) cells and KSHV-transformed MM (KMM) cells [77]. Multiple TLRs were upregulated in KMM cells compared to MM cells [77]. Notably, TLR4 was upregulated by over 40 times in KMM cells compared to MM cells [77]. Authors also observed that TLR4 was upregulated in KSHV-infected spindle tumor cells in human KS lesions and KSHV-infected TIME cells [77]. Mechanistically, Gruffaz et al. reported that KSHV-upregulated expression of TLR4 was mediated by multiple viral miRNAs, and that activation of the TLR4 pathway in KSHV-infected cells results in chronic induction of cytokines IL6, IL1 $\beta$, and IL18 [77]. The study also demonstrated that IL6 mediated constitutive activation of the STAT3 pathway [77]. These results emphasized the essentiality of TLR4 pathway in KSHV-induced cellular transformation and tumorigenesis, underscoring the impact of bacterial infection in exacerbating KSHV-induced pathology.

Markazi et al. further investigated the effects of TLR4 by stimulating MM/KMM and BJAB/KSHV-infected BJAB cells (EBV-negative B-cell lymphoma cells) with Pseudomonas aeruginosa [78]. P. aeruginosa is a gram-negative opportunistic pathogen that can infect immunosuppressed individuals [79]. P. aeruginosa infection resulted in increased proliferation in KSHV-infected BJAB and KMM cells but had no significant effects in the KSHV uninfected control cells [78]. As KMM cells have previously been shown to form colonies in soft agar [80], Markazi et al. demonstrated that P. aeruginosa stimulation increased cell proliferation and efficiency of colony formation in soft agar of KMM cells but had no significant effect on the untransformed MM cells [78]. Mechanistically, the study showed that P. aeruginosa increased inflammatory cytokines and activation of p38, ERK1/2, and JNK MAPK pathways in KMM cells through LPS and flagellin ligands [78]. These results imply that opportunistic infection is likely an important target in mitigating KS pathology. Furthermore, this study shows that it is necessary to assess individual bacterial PAMPs on the activation of cell signaling pathways in order to more effectively elucidate molecules involved in inflammation and identify the potential therapeutic targets for KSHV-induced cancers. In this study, LPS and flagellin ligands were assessed for their effects on inducing inflammation in KSHV-infected cells. However, bacteria possess many other PAMPs (lipoproteins, LTA, peptidoglycans, etc.) and secrete by-products $\left(\mathrm{H}_{2} \mathrm{O}_{2}\right.$, SCFAs, quorum signaling molecules, etc.) that may induce proliferation in KSHV-latently infected cells, and therefore may be worth further investigation.

\section{Conclusions and Future Perspectives}

The collection of studies reviewed herein has demonstrated that bacteria can affect KSHV-induced cancers by numerous mechanisms, including through bacterial PAMPS and bacterial-secreted byproducts. Indeed, microbiome analyses reveal increased levels of pathogenic bacteria and secreted PAMPs in immunosuppressed HIV / KSHV-coinfected patients $[51,54,67]$. Bacteria can reactivate KSHV through both direct and indirect methods. Bacterial species such as F. nucleatum and P. gingivalis produce SCFAs which suppress HDAC activity, resulting in increased hyperacetylation and subsequent expression of lytic gene expression $[53,55,56]$. Bacteria can also induce inflammation through PAMPs which may reactivate KSHV indirectly through increased ROS production $[63,64,67]$. KSHV reactivation leads to the spread and dissemination of the virus [40]. Moreover, recent studies have demonstrated that inflammation induced by bacterial LPS and flagellin PAMPs may enhance cell proliferation and KSHV-induced cellular transformation in the latently infected 
cells $[77,78]$. Analyzing the effects of bacteria on latently infected cells is especially relevant since the majority of KSHV-infected cells within KS tumors are in the latent state [72]. By elucidating the various mechanisms of bacteria-induced effects on KSHV-infected cells, researchers gain important insights into the cell signaling pathways involved in KSHV replication and dissemination as well as increased survival and proliferation of tumor cells.

Since interactions among bacterial species and the host are complex, it is crucial to more thoroughly investigate the microbial signature of HIV / KSHV-coinfected patients to enhance our understanding of bacterial biomarkers and the underlying mechanisms that contribute to KSHV pathology. Many studies have reported that immunosuppression and inflammation drive KSHV pathology, both of which are applicable to bacterial infection [4]. Interestingly, there is a paucity of data assessing the effects of KSHV infection on microbiome dysregulation. However, since KSHV encodes multiple proteins (e.g., K3, K5, LANA, and RTA) that interfere with the host immune response, it can be speculated that KSHV might alter the host microbiome by deregulating immune response [81].

While the majority of microbiome analyses in HIV/KSHV-coinfected patients used saliva samples, it may be beneficial to assess the microbiome in additional sites, such as KS lesions present on different organs (skin, lungs, intestines, lymph nodes, etc.) as microbial species vary depending where they are located [31]. Moreover, while studies have reported higher levels of bacterial PAMPs in immunosuppressed patients, including LPS, LTA, and flagellin, the kits used to analyze these PAMPs do not distinguish between different bacterial species [19,67]. There is evidence that the same types of PAMPs (i.e., LPS) from different bacterial species can have varying effects on the immune responses [71]. Hence, it might be important to delineate the distinct effects of specific bacterial species on KSHVinduced cancers.

It has been demonstrated that the NF- $\mathrm{KB}$, STAT3, and MAPK pathways play crucial roles in KSHV reactivation and cell proliferation of KSHV latently infected cells [55,63,77,78]. It can be speculated that activation of MAPK pathways by bacteria can increase KSHV infectivity and viral dissemination $[57,59,60]$ while bacteria-induced inflammation can also promote tumorigenesis through the STAT3 pathway [77]. However, as there are numerous PRRs and downstream cascades associated with bacterial infection, it is highly likely that additional pathways could be involved in promoting KS pathology. The effects of bacteria on the immune response are highly intricate, and pathways including cytoplasmic bacterial immune sensors such as nod-like receptors or the cGAS-STING pathway have not yet been explored in relation to bacterial effects on KSHV infection and KSHV-induced cell proliferation $[6,82]$. Furthermore, although the studies presented in this review examine the activation of pro-survival pathways by bacterial PAMPs and metabolic products, there are additional bacterial products that may activate similar pathways in KSHV-infected cells. For example, several studies report that bacterial biofilms and quorum signaling molecules can induce inflammatory immune responses; hence it may be worth investigating their effects on promoting KSHV-induced cancers [83-85]. Thorough understanding of bacterial PAMPs and associated cell signaling pathways involved in KSHV pathogenesis is essential for discovering novel molecular targets for therapeutic intervention of KSHV-induced cancers.

The utilization of KSHV-infected cells in cell culture experiments has been proven invaluable for providing data on the effects of bacteria on KS pathology. The KSHVinfected cells used in both the Gruffaz et al. and Markazi et al. studies were developed by infecting primary rat embryonic metanephric mesenchymal precursor cells $[77,78,80]$. This resulted in complete cellular transformation characterized by immortalization, colony formation in soft agar, and tumor induction in mice [80]. Although in vitro experiments have revealed that bacteria can regulate cell proliferation and viral replication of KSHVinfected cells, the effect of bacteria on promoting KSHV infection and the process of cellular transformation have not yet been examined. Since KSHV-associated bacteria can activate mitogenic pathways necessary for KSHV primary infection, it is plausible that bacteria might promote cellular transformation as well $[57,77,78]$. 
The collection of studies explored in this review focus predominantly on pathogenic bacteria enhancing KSHV-induced cancer pathology. However, bacteria explored as anticancer therapies have also been of interest for multiple cancer types [25,26]. Moreover, abundant evidence demonstrates that certain bacterial species (e.g., probiotics) can mitigate cancer pathology [3]. Probiotics may enhance immunity against cancer through multiple mechanisms including out competition of pathogenic bacteria, degradation of carcinogens, and production of anti-inflammatory mediators [3]. Although Gruffaz et al. reported increased levels of the Firmicutes phylum in KS patient, Firmicutes bacteria are potent producers of SCFA such as butyrate, which can reactivate KSHV and other herpesviruses $[51,56,86]$. This feature of Firmicutes bacteria potentially complicates the use of probiotic bacteria such as Lactobacillus as "anti-inflammatory" bacteria as they can be potent producers of SCFAs [3]. Further inquiry into the effects of therapeutic bacteria on KSHV-driven diseases may be critical in future studies.

Notwithstanding the potential of anti-tumor bacterial species, the studies explored in this review strongly emphasize the negative effects of pathogenic, opportunistic infection in patients with KSHV-induced cancers. As these cancers continue to be the most common cancer types in immunosuppressed patients despite increased use of antiretroviral drugs, it is evident that more efficient treatments are in demand [37]. Microbiome research in relation to KSHV-induced cancers is undoubtedly a crucial step in pioneering enhanced therapies for mitigating KSHV pathology.

Author Contributions: Conceptualization, S.-J.G.; writing—original draft preparation, A.M.; writingreview and editing, A.M., W.M., P.M.B., M.S.M. and S.-J.G.; visualization, A.M.; project administration and funding acquisition, S.-J.G. All authors have read and agreed to the published version of the manuscript.

Funding: This research was funded by National Institute of Health, grant numbers CA096512, CA124332, CA132637, CA213275, CA177377, DE025465 and CA197153, to S.J.G. W.M. was in part supported by the AIDS and Cancer Specimen Resource (ACSR) Young Investigator Pilot Award (YIPA), funded by the National Cancer Institute.

Conflicts of Interest: The authors declare no conflict of interest.

\section{References}

1. Bhatt, A.P.; Redinbo, M.R.; Bultman, S.J. The role of the microbiome in cancer development and therapy. CA Cancer J. Clin. 2017, 67, 326-344. [CrossRef]

2. van Elsland, D.; Neefjes, J. Bacterial infections and cancer. EMBO Rep. 2018, 19. [CrossRef]

3. Górska, A.; Przystupski, D.; Niemczura, M.J.; Kulbacka, J. Probiotic bacteria: A promising tool in cancer prevention and therapy. Curr. Microbiol. 2019, 76, 939-949. [CrossRef]

4. Thakker, S.; Verma, S.C. Co-infections and pathogenesis of KSHV-associated malignancies. Front. Microbiol. $2016,7,151$. [CrossRef]

5. Morales-Sánchez, A.; Fuentes-Pananá, E.M. Human viruses and cancer. Viruses 2014, 6, 4047-4079. [CrossRef]

6. Mogensen, T.H. Pathogen recognition and inflammatory signaling in innate immune defenses. Clin. Microbiol. Rev. 2009, 22, 240-273. [CrossRef]

7. Kawai, T.; Akira, S. The roles of TLRs, RLRs and NLRs in pathogen recognition. Int. Immunol. 2009, 21, 317-337. [CrossRef]

8. Coussens, L.M.; Werb, Z. Inflammation and cancer. Nature 2002, 420, 860-867. [CrossRef] [PubMed]

9. Markiewski, M.M.; Lambris, J.D. The role of complement in inflammatory diseases from behind the scenes into the spotlight. Am. J. Pathol. 2007, 171, 715-727. [CrossRef]

10. Singh, N.; Baby, D.; Rajguru, J.P.; Patil, P.B.; Thakkannavar, S.S.; Pujari, V.B. Inflammation and cancer. Ann. Afr. Med. 2019, 18, 121-126. [CrossRef]

11. Qu, X.; Tang, Y.; Hua, S. Immunological approaches towards cancer and inflammation: A cross talk. Front. Immunol. 2018, 9, 563. [CrossRef]

12. Kawasaki, T.; Kawai, T. Toll-like receptor signaling pathways. Front. Immunol. 2014, 5, 461. [CrossRef]

13. Hoving, J.C.; Wilson, G.J.; Brown, G.D. Signalling C-type lectin receptors, microbial recognition and immunity. Cell Microbiol. 2014, 16, 185-194. [CrossRef] [PubMed]

14. Yang, H.; Wang, B.; Wang, T.; Xu, L.; He, C.; Wen, H.; Yan, J.; Su, H.; Zhu, X. Toll-like receptor 4 prompts human breast cancer cells invasiveness via lipopolysaccharide stimulation and is overexpressed in patients with lymph node metastasis. PLoS ONE 2014, 9, e109980. [CrossRef] [PubMed] 
15. Li, N.; Xu, H.; Ou, Y.; Feng, Z.; Zhang, Q.; Zhu, Q.; Cai, Z. LPS-induced CXCR7 expression promotes gastric Cancer proliferation and migration via the TLR4/MD-2 pathway. Diagn. Pathol. 2019, 14, 3. [CrossRef] [PubMed]

16. Song, E.J.; Kang, M.J.; Kim, Y.S.; Kim, S.M.; Lee, S.E.; Kim, C.H.; Kim, D.J.; Park, J.H. Flagellin promotes the proliferation of gastric cancer cells via the Toll-like receptor 5. Int. J. Mol. Med. 2011, 28, 115-119. [CrossRef] [PubMed]

17. Xie, W.; Huang, Y.; Xie, W.; Guo, A.; Wu, W. Bacteria peptidoglycan promoted breast cancer cell invasiveness and adhesiveness by targeting toll-like receptor 2 in the cancer cells. PLOS ONE 2010, 5, e10850. [CrossRef]

18. Jain, S.; Dash, P.; Minz, A.P.; Satpathi, S.; Samal, A.G.; Behera, P.K.; Satpathi, P.S.; Senapati, S. Lipopolysaccharide (LPS) enhances prostate cancer metastasis potentially through NF- $\mathrm{kB}$ activation and recurrent dexamethasone administration fails to suppress it in vivo. Prostate 2019, 79, 168-182. [CrossRef] [PubMed]

19. Fedirko, V.; Tran, H.Q.; Gewirtz, A.T.; Stepien, M.; Trichopoulou, A.; Aleksandrova, K.; Olsen, A.; Tjønneland, A.; Overvad, K.; Carbonnel, F.; et al. Exposure to bacterial products lipopolysaccharide and flagellin and hepatocellular carcinoma: A nested case-control study. BMC Med. 2017, 15, 72. [CrossRef]

20. Kong, S.Y.; Tran, H.Q.; Gewirtz, A.T.; McKeown-Eyssen, G.; Fedirko, V.; Romieu, I.; Tjønneland, A.; Olsen, A.; Overvad, K.; Boutron-Ruault, M.C.; et al. Serum endotoxins and flagellin and risk of colorectal cancer in the European prospective investigation into cancer and nutrition (EPIC) cohort. Cancer Epidemiol. Biomark. Prev. 2016, 25, 291-301. [CrossRef]

21. de Waal, G.M.; de Villiers, W.J.S.; Forgan, T.; Roberts, T.; Pretorius, E. Colorectal cancer is associated with increased circulating lipopolysaccharide, inflammation and hypercoagulability. Sci. Rep. 2020, 10, 8777. [CrossRef]

22. Shi, J.; Zhao, Y.; Wang, Y.; Gao, W.; Ding, J.; Li, P.; Hu, L.; Shao, F. Inflammatory caspases are innate immune receptors for intracellular LPS. Nature 2014, 514, 187-192. [CrossRef]

23. Fried, S.; Tosun, S.; Troost, G.; Keil, S.; Zaenker, K.S.; Dittmar, T. Lipopolysaccharide (LPS) promotes apoptosis in human breast epithelial $\times$ breast cancer hybrids, but not in parental cells. PLoS ONE 2016, 11, e0148438. [CrossRef]

24. Cai, Z.; Sanchez, A.; Shi, Z.; Zhang, T.; Liu, M.; Zhang, D. Activation of Toll-like receptor 5 on breast cancer cells by flagellin suppresses cell proliferation and tumor growth. Cancer Res. 2011, 71, 2466-2475. [CrossRef]

25. Duong, M.T.; Qin, Y.; You, S.H.; Min, J.J. Bacteria-cancer interactions: Bacteria-based cancer therapy. Exp. Mol. Med. 2019, 51, 1-15. [CrossRef]

26. Shetab Boushehri, M.A.; Lamprecht, A. Challenges of using lipopolysaccharides for cancer immunotherapy and potential delivery-based solutions thereto. Ther. Deliv. 2019, 10, 165-187. [CrossRef]

27. Jouhi, L.; Renkonen, S.; Atula, T.; Mäkitie, A.; Haglund, C.; Hagström, J. Different toll-like receptor expression patterns in progression toward cancer. Front. Immunol. 2014, 5, 638. [CrossRef]

28. Khan, A.A.; Khan, Z.; Warnakulasuriya, S. Cancer-associated toll-like receptor modulation and insinuation in infection susceptibility: Association or coincidence? Ann. Oncol. 2016, 27, 984-997. [CrossRef] [PubMed]

29. Binnewies, M.; Roberts, E.W.; Kersten, K.; Chan, V.; Fearon, D.F.; Merad, M.; Coussens, L.M.; Gabrilovich, D.I.; Ostrand-Rosenberg, S.; Hedrick, C.C.; et al. Understanding the tumor immune microenvironment (TIME) for effective therapy. Nat. Med. 2018, 24, 541-550. [CrossRef]

30. Berg, G.; Rybakova, D.; Fischer, D.; Cernava, T.; Vergès, M.C.; Charles, T.; Chen, X.; Cocolin, L.; Eversole, K.; Corral, G.H.; et al. Microbiome definition re-visited: Old concepts and new challenges. Microbiome 2020, 8, 103. [CrossRef]

31. Ursell, L.K.; Metcalf, J.L.; Parfrey, L.W.; Knight, R. Defining the human microbiome. Nutr. Rev. 2012, 70, S38-S44. [CrossRef] [PubMed]

32. Nejman, D.; Livyatan, I.; Fuks, G.; Gavert, N.; Zwang, Y.; Geller, L.T.; Rotter-Maskowitz, A.; Weiser, R.; Mallel, G.; Gigi, E.; et al. The human tumor microbiome is composed of tumor type-specific intracellular bacteria. Science 2020, 368, 973-980. [CrossRef] [PubMed]

33. Belkaid, Y.; Hand, T.W. Role of the microbiota in immunity and inflammation. Cell 2014, 157, 121-141. [CrossRef] [PubMed]

34. Cho, I.; Blaser, M.J. The human microbiome: At the interface of health and disease. Nat. Rev. Genet. 2012, 13, 260-270. [CrossRef] [PubMed]

35. He, M.; Cheng, F.; da Silva, S.R.; Tan, B.; Sorel, O.; Gruffaz, M.; Li, T.; Gao, S.J. Molecular biology of KSHV in relation to HIV / AIDS-associated oncogenesis. Cancer Treat. Res. 2019, 177, 23-62. [CrossRef]

36. Osmond, D.H.; Buchbinder, S.; Cheng, A.; Graves, A.; Vittinghoff, E.; Cossen, C.K.; Forghani, B.; Martin, J.N. Prevalence of Kaposi sarcoma-associated herpesvirus infection in homosexual men at beginning of and during the HIV epidemic. JAMA 2002, 287, 221-225. [CrossRef]

37. Cesarman, E.; Damania, B.; Krown, S.E.; Martin, J.; Bower, M.; Whitby, D. Kaposi sarcoma. Nat. Rev. Dis. Primers 2019, 5, 9. [CrossRef]

38. Bhutani, M.; Polizzotto, M.N.; Uldrick, T.S.; Yarchoan, R. Kaposi sarcoma-associated herpesvirus-associated malignancies: Epidemiology, pathogenesis, and advances in treatment. Semin. Oncol. 2015, 42, 223-246. [CrossRef]

39. Moore, P.S.; Chang, Y. Why do viruses cause cancer? Highlights of the first century of human tumour virology. Nat. Rev. Cancer 2010, 10, 878-889. [CrossRef]

40. Ye, F.; Lei, X.; Gao, S.J. Mechanisms of Kaposi's sarcoma-associated herpesvirus latency and reactivation. Adv. Virol. 2011, 2011, 193860. [CrossRef]

41. Okoye, A.A.; Picker, L.J. CD4(+) T-cell depletion in HIV infection: Mechanisms of immunological failure. Immunol. Rev. 2013, 254, 54-64. [CrossRef] 
42. Aneja, K.K.; Yuan, Y. Reactivation and lytic replication of Kaposi's sarcoma-associated herpesvirus: An update. Front. Microbiol. 2017, 8, 613. [CrossRef]

43. Yan, Q.; Shen, C.; Qin, J.; Li, W.; Hu, M.; Lu, H.; Qin, D.; Zhu, J.; Gao, S.J.; Lu, C. HIV-1 Vpr inhibits Kaposi's sarcoma-associated herpesvirus lytic replication by inducing microRNA miR-942-5p and activating NF-кB signaling. J. Virol. 2016, 90, 8739-8753. [CrossRef]

44. Yao, S.; Hu, M.; Hao, T.; Li, W.; Xue, X.; Xue, M.; Zhu, X.; Zhou, F.; Qin, D.; Yan, Q.; et al. MiRNA-891a-5p mediates HIV-1 Tat and KSHV Orf-K1 synergistic induction of angiogenesis by activating NF- $\mathrm{kB}$ signaling. Nucleic Acids Res. 2019, 47, 2700. [CrossRef]

45. Yan, Q.; Zhao, R.; Shen, C.; Wang, F.; Li, W.; Gao, S.J.; Lu, C. Upregulation of microRNA 711 mediates HIV-1 Vpr promotion of Kaposi's sarcoma-associated herpesvirus latency and induction of pro-proliferation and pro-survival cytokines by targeting the Notch/NF-kB-signaling axis. J. Virol. 2018, 92, e00580-18. [CrossRef]

46. Zhou, F.; Xue, M.; Qin, D.; Zhu, X.; Wang, C.; Zhu, J.; Hao, T.; Cheng, L.; Chen, X.; Bai, Z.; et al. HIV-1 Tat promotes Kaposi's sarcoma-associated herpesvirus (KSHV) vIL-6-induced angiogenesis and tumorigenesis by regulating PI3K/PTEN/AKT/GSK-3 $\beta$ signaling pathway. PLoS ONE 2013, 8, e53145. [CrossRef]

47. Zeng, Y.; Zhang, X.; Huang, Z.; Cheng, L.; Yao, S.; Qin, D.; Chen, X.; Tang, Q.; Lv, Z.; Zhang, L.; et al. Intracellular Tat of human immunodeficiency virus type 1 activates lytic cycle replication of Kaposi's sarcoma-associated herpesvirus: Role of JAK/STAT signaling. J. Virol. 2007, 81, 2401-2417. [CrossRef]

48. Xue, M.; Yao, S.; Hu, M.; Li, W.; Hao, T.; Zhou, F.; Zhu, X.; Lu, H.; Qin, D.; Yan, Q.; et al. HIV-1 Nef and KSHV oncogene K1 synergistically promote angiogenesis by inducing cellular miR-718 to regulate the PTEN/AKT/mTOR signaling pathway. Nucleic Acids Res. 2014, 42, 9862-9879. [CrossRef]

49. Annavajhala, M.K.; Khan, S.D.; Sullivan, S.B.; Shah, J.; Pass, L.; Kister, K.; Kunen, H.; Chiang, V.; Monnot, G.C.; Ricupero, C.L.; et al. Oral and gut microbial diversity and immune regulation in patients with HIV on antiretroviral therapy. $m S p h e r e$ 2020, 5, e00798-19. [CrossRef]

50. Taur, Y.; Pamer, E.G. The intestinal microbiota and susceptibility to infection in immunocompromised patients. Curr. Opin. Infect. Dis. 2013, 26, 332-337. [CrossRef]

51. Gruffaz, M.; Zhang, T.; Marshall, V.; Gonçalves, P.; Ramaswami, R.; Labo, N.; Whitby, D.; Uldrick, T.S.; Yarchoan, R.; Huang, Y.; et al. Signatures of oral microbiome in HIV-infected individuals with oral Kaposi's sarcoma and cell-associated KSHV DNA. PLoS Pathog. 2020, 16, e1008114. [CrossRef]

52. Contreras, A.; Slots, J. Herpesviruses in human periodontal disease. J. Periodontal. Res. 2000, 35, 3-16. [CrossRef]

53. Yu, X.; Shahir, A.M.; Sha, J.; Feng, Z.; Eapen, B.; Nithianantham, S.; Das, B.; Karn, J.; Weinberg, A.; Bissada, N.F.; et al. Short-chain fatty acids from periodontal pathogens suppress histone deacetylases, EZH2, and SUV39H1 to promote Kaposi's sarcoma-associated herpesvirus replication. J. Virol. 2014, 88, 4466-4479. [CrossRef] [PubMed]

54. Dai, L.; Barrett, L.; Plaisance-Bonstaff, K.; Post, S.R.; Qin, Z. Porphyromonas gingivalis coinfects with KSHV in oral cavities of HIV+ patients and induces viral lytic reactivation. J. Med. Virol. 2020, 92, 3862-3867. [CrossRef]

55. Morris, T.L.; Arnold, R.R.; Webster-Cyriaque, J. Signaling cascades triggered by bacterial metabolic end products during reactivation of Kaposi's sarcoma-associated herpesvirus. J. Virol. 2007, 81, 6032-6042. [CrossRef]

56. Davie, J.R. Inhibition of histone deacetylase activity by butyrate. J. Nutr. 2003, 133, 2485s-2493s. [CrossRef] [PubMed]

57. Pan, H.; Xie, J.; Ye, F.; Gao, S.J. Modulation of Kaposi's sarcoma-associated herpesvirus infection and replication by MEK/ERK, JNK, and p38 multiple mitogen-activated protein kinase pathways during primary infection. J. Virol. 2006, 80, 5371-5382. [CrossRef] [PubMed]

58. Lei, X.; Bai, Z.; Ye, F.; Xie, J.; Kim, C.G.; Huang, Y.; Gao, S.J. Regulation of NF-kappaB inhibitor IkappaBalpha and viral replication by a KSHV microRNA. Nat. Cell Biol. 2010, 12, 193-199. [CrossRef]

59. Xie, J.; Pan, H.; Yoo, S.; Gao, S.J. Kaposi's sarcoma-associated herpesvirus induction of AP-1 and interleukin 6 during primary infection mediated by multiple mitogen-activated protein kinase pathways. J. Virol. 2005, 79, 15027-15037. [CrossRef]

60. Xie, J.; Ajibade, A.O.; Ye, F.; Kuhne, K.; Gao, S.J. Reactivation of Kaposi's sarcoma-associated herpesvirus from latency requires MEK/ERK, JNK and p38 multiple mitogen-activated protein kinase pathways. Virology 2008, 371, 139-154. [CrossRef] [PubMed]

61. Ye, F.; Gao, S.J. A novel role of hydrogen peroxide in Kaposi sarcoma-associated herpesvirus reactivation. Cell Cycle 2011, 10, 3237-3238. [CrossRef]

62. Erttmann, S.F.; Gekara, N.O. Hydrogen peroxide release by bacteria suppresses inflammasome-dependent innate immunity. Nat. Commun. 2019, 10, 3493. [CrossRef]

63. Dai, L.; DeFee, M.R.; Cao, Y.; Wen, J.; Wen, X.; Noverr, M.C.; Qin, Z. Lipoteichoic acid (LTA) and lipopolysaccharides (LPS) from periodontal pathogenic bacteria facilitate oncogenic herpesvirus infection within primary oral cells. PLoS ONE 2014, 9, e101326. [CrossRef] [PubMed]

64. Bottero, V.; Chakraborty, S.; Chandran, B. Reactive oxygen species are induced by Kaposi's sarcoma-associated herpesvirus early during primary infection of endothelial cells to promote virus entry. J. Virol. 2013, 87, 1733-1749. [CrossRef]

65. Sharma-Walia, N.; Krishnan, H.H.; Naranatt, P.P.; Zeng, L.; Smith, M.S.; Chandran, B. ERK1/2 and MEK1/2 induced by Kaposi's sarcoma-associated herpesvirus (human herpesvirus 8) early during infection of target cells are essential for expression of viral genes and for establishment of infection. J. Virol. 2005, 79, 10308-10329. [CrossRef] [PubMed] 
66. Sadagopan, S.; Sharma-Walia, N.; Veettil, M.V.; Raghu, H.; Sivakumar, R.; Bottero, V.; Chandran, B. Kaposi's sarcoma-associated herpesvirus induces sustained NF-kappaB activation during de novo infection of primary human dermal microvascular endothelial cells that is essential for viral gene expression. J. Virol. 2007, 81, 3949-3968. [CrossRef]

67. Dai, L.; Qiao, J.; Yin, J.; Goldstein, A.; Lin, H.Y.; Post, S.R.; Qin, Z. Kaposi sarcoma-associated herpesvirus and Staphylococcus aureus coinfection in oral cavities of HIV-positive patients: A unique niche for oncogenic virus lytic reactivation. J. Infect. Dis. 2020, 221, 1331-1341. [CrossRef] [PubMed]

68. Cuesta, A.I.; Jewtuchowicz, V.; Brusca, M.I.; Nastri, M.L.; Rosa, A.C. Prevalence of Staphylococcus spp. and Candida spp. in the oral cavity and periodontal pockets of periodontal disease patients. Acta Odontol. Lat. 2010, 23, 20-26.

69. Ferreira Dde, C.; Silva, G.R.; Cavalcante, F.S.; Carmo, F.L.; Fernandes, L.A.; Moreira, S.; Passos, M.R.; Colombo, A.P.; Santos, K.R. Methicillin-resistant Staphylococcus aureus in HIV patients: Risk factors associated with colonization and/or infection and methods for characterization of isolates-A systematic review. Clinics 2014, 69, 770-776. [CrossRef] [PubMed]

70. Tribble, G.D.; Kerr, J.E.; Wang, B.Y. Genetic diversity in the oral pathogen Porphyromonas gingivalis: Molecular mechanisms and biological consequences. Future Microbiol. 2013, 8, 607-620. [CrossRef]

71. Bertani, B.; Ruiz, N. Function and Biogenesis of Lipopolysaccharides. EcoSal Plus 2018, 8, 1. [CrossRef] [PubMed]

72. Giffin, L.; Damania, B. KSHV: Pathways to tumorigenesis and persistent infection. Adv. Virus Res. 2014, 88, 111-159. [CrossRef]

73. Campbell, M.; Yang, W.S.; Yeh, W.W.; Kao, C.H.; Chang, P.C. Epigenetic regulation of Kaposi's sarcoma-associated herpesvirus latency. Front. Microbiol. 2020, 11, 850. [CrossRef]

74. Punjabi, A.S.; Carroll, P.A.; Chen, L.; Lagunoff, M. Persistent activation of STAT3 by latent Kaposi's sarcoma-associated herpesvirus infection of endothelial cells. J. Virol. 2007, 81, 2449-2458. [CrossRef]

75. King, C.A.; Li, X.; Barbachano-Guerrero, A.; Bhaduri-McIntosh, S. STAT3 regulates lytic activation of Kaposi's sarcoma-associated herpesvirus. J. Virol. 2015, 89, 11347-11355. [CrossRef] [PubMed]

76. Lee, M.S.; Jones, T.; Song, D.Y.; Jang, J.H.; Jung, J.U.; Gao, S.J. Exploitation of the complement system by oncogenic Kaposi's sarcoma-associated herpesvirus for cell survival and persistent infection. PLoS Pathog. 2014, 10, e1004412. [CrossRef] [PubMed]

77. Gruffaz, M.; Vasan, K.; Tan, B.; Ramos da Silva, S.; Gao, S.J. TLR4-mediated inflammation promotes KSHV-induced cellular transformation and tumorigenesis by activating the STAT3 pathway. Cancer Res. 2017, 77, 7094-7108. [CrossRef] [PubMed]

78. Markazi, A.; Bracci, P.M.; McGrath, M.; Gao, S.J. Pseudomonas aeruginosa stimulates inflammation and enhances Kaposi's sarcoma herpesvirus-induced cell proliferation and cellular transformation through both lipopolysaccharide and flagellin. $m B i o$ 2020, 11, e02843-20. [CrossRef] [PubMed]

79. Sadikot, R.T.; Blackwell, T.S.; Christman, J.W.; Prince, A.S. Pathogen-host interactions in Pseudomonas aeruginosa pneumonia. Am. J. Respir. Crit. Care Med. 2005, 171, 1209-1223. [CrossRef]

80. Jones, T.; Ye, F.; Bedolla, R.; Huang, Y.; Meng, J.; Qian, L.; Pan, H.; Zhou, F.; Moody, R.; Wagner, B.; et al. Direct and efficient cellular transformation of primary rat mesenchymal precursor cells by KSHV. J. Clin. Investig. 2012, 122, 1076-1081. [CrossRef] [PubMed]

81. Broussard, G.; Damania, B. KSHV: Immune modulation and immunotherapy. Front. Immunol. 2020, 10, 3084. [CrossRef] [PubMed]

82. Ma, Z.; Jacobs, S.R.; West, J.A.; Stopford, C.; Zhang, Z.; Davis, Z.; Barber, G.N.; Glaunsinger, B.A.; Dittmer, D.P.; Damania, B. Modulation of the cGAS-STING DNA sensing pathway by gammaherpesviruses. Proc. Natl. Acad. Sci. USA 2015, 112, E4306-E4315. [CrossRef] [PubMed]

83. Wynendaele, E.; Verbeke, F.; D’Hondt, M.; Hendrix, A.; Van De Wiele, C.; Burvenich, C.; Peremans, K.; De Wever, O.; Bracke, M.; De Spiegeleer, B. Crosstalk between the microbiome and cancer cells by quorum sensing peptides. Peptides 2015, 64, 40-48. [CrossRef] [PubMed]

84. Grabiner, M.A.; Fu, Z.; Wu, T.; Barry, K.C.; Schwarzer, C.; Machen, T.E. Pseudomonas aeruginosa quorum-sensing molecule homoserine lactone modulates inflammatory signaling through PERK and eI-F2 $\alpha$. J. Immunol. 2014, 193, 1459-1467. [CrossRef] [PubMed]

85. Qiao, J.; Cao, Y.; Zabaleta, J.; Yang, L.; Dai, L.; Qin, Z. Regulation of virus-associated lymphoma growth and gene expression by bacterial quorum-sensing molecules. J. Virol. 2018, 92, e00478-18. [CrossRef]

86. Louis, P.; Flint, H.J. Formation of propionate and butyrate by the human colonic microbiota. Environ. Microbiol. 2017, 19, 29-41. [CrossRef] 